\title{
Effect of periodontal therapy on COPD outcomes: a systematic review
}

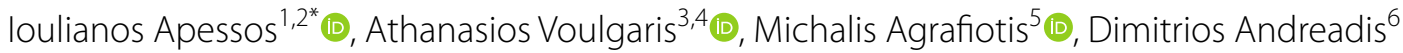 \\ and Paschalis Steiropoulos 3,4
}

\begin{abstract}
Background: Latest evidence suggests that periodontitis is prevalent among patients with chronic obstructive pulmonary disease (COPD), while recent studies have also reported a potential benefit of periodontal treatment on several COPD outcomes. This systematic review aims to determine the impact of periodontal treatment on exacerbation rate, lung function and quality of life of COPD patients.

Methods: A systematic search of electronic databases of PubMed, Scopus, Virtual Health Library, ScienceDirect, Wiley Online Library, Web of Science, ProQuest Dissertation and Theses Global and Google Scholar was conducted. Search restricted to studies involving human subjects which were published from January 2000 to March 2020 in English language. Distiller Systematic Review software was used for data management. Risk of bias was assessed using Risk of Bias 2 (RoB2) and Risk of Bias for non-randomized studies of intervention (ROBINS-I) tools. Overall quality of evidence was judged based on Grading of Recommendations Assessment, Development and Evaluation working group methodology.
\end{abstract}

Results: Out of 1442 articles retrieved, 7 full text articles were included in the review. Limited evidence suggests that periodontal treatment in patients with COPD and periodontitis is associated with reduced exacerbation frequency and a slower lung function decline rate, while its effects on quality of life remain unclear. In addition, periodontal treatment in COPD is associated with lower hospitalization rates and reduced all-cause mortality. Significant methodological differences were noted amongst included studies, while very low-to-moderate overall quality of evidence was demonstrated.

Conclusions: Although it is reasonable to advise COPD patients not to neglect their dental health, further studies are warranted to determine the role of periodontal therapy on COPD clinical outcomes.

Trial Registration: PROSPERO 2020 (CRD42020158481). https://www.crd.york.ac.uk/prospero/display_recor d.php?|D=CRD42020158481

Keywords: Chronic obstructive pulmonary disease, COPD exacerbation, Periodontal therapy, Oral health, Systematic review

*Correspondence: julianapessos@hotmail.com

${ }^{1}$ Department of Oral Surgery, Surgical Implantology and Oral Radiology,

School of Health Sciences, School of Dentistry, Aristotle University

of Thessaloniki, 54124 Thessaloniki, Greece

Full list of author information is available at the end of the article

\section{Background}

Chronic obstructive pulmonary disease (COPD) is characterized by the presence of partially reversible airflow limitation with associated symptoms and history compatible with exposure to tobacco and other noxious particles or gases [1]. COPD is a common, preventable and treatable disease and constitutes a major 
cause of morbidity and mortality [1] with an increasing burden placed the $3^{\text {rd }}$ leading cause of death in 2020 [2]. COPD exacerbations are complex events, triggered by respiratory viral or bacterial pathogens, as well as environmental factors such as pollution and ambient temperature, and often require treatment changes or even hospital admission. In addition, they can negatively impact on health status, hospitalization rates and disease progression and ultimately result in poorer outcomes [3, 4].

COPD often coexists with several diseases that may significantly affect patients' prognosis [5]. Cardiovascular disease (CVD), hypertension, lung cancer, metabolic syndrome, and obstructive sleep apnea are among the most prevalent comorbidities in COPD patients [5, 6]. Periodontitis is a chronic multifactorial inflammatory disease associated with dysbiotic plaque biofilms and is characterized by progressive destruction of the tooth supporting tissue [7]. Periodontitis can result in tooth loss, while it may also affect chewing function and aesthetics, leading ultimately to impaired quality of life. Moreover, periodontitis accounts for a substantial proportion of edentulism and masticatory dysfunction cases, resulting in significant dental care costs [7]. Overall, periodontitis exerts a major burden on public health systems [8].

Periodontitis and COPD are chronic progressive inflammatory diseases that share common risk factors such as smoking, age and lower socioeconomic status $[9,10]$. Evolving evidence points towards an association between COPD, oral health and periodontal disease [8]. In a meta-analysis of 14 observational studies, the presence of periodontitis had an odds ratio (OR) of 2.08 for developing COPD [11]. On the other hand, when compared to the general population, COPD patients had a 1.2 higher risk for developing periodontal disease, which increased to 3.17 for those with prior hospitalization [12]; additionally the presence of periodontal disease is associated with worse pulmonary function in the COPD population [13, 14].

Based on the results of the abovementioned studies one might argue that routine assessment and treatment of periodontal disease could exert a beneficial effect on COPD clinical and functional outcomes. However, while previous systematic reviews have investigated the relationship between periodontitis and COPD, no recent studies have reported on the role of periodontal treatment of COPD outcomes [11, 15-17]. Therefore, the aim of the present study is to systematically review the most recent evidence on the effect of non-surgical or surgical periodontal therapy on quality of life, lung function and exacerbations of patients with COPD and periodontitis.

\section{Methods}

\section{Protocol and registration}

This review was registered in the center for Reviews and Dissemination, University of York, (protocol number: UK \#CRD42020158481). The inclusion/exclusion criteria were specified a priori and followed the PICO criteria and Preferred Reporting Items for Systematic Reviews and Meta-Analyses protocol (PRISMA-P) guidelines [18].

\section{Information sources and search strategy}

The following databases were searched by one author (IA) using a combination of keywords for COPD, periodontitis and periodontal therapy: PubMed, Scopus, Virtual Health Library, ScienceDirect, Wiley Online Library, Web of Science, ProQuest Dissertation and Theses Global and Google Scholar (Table 1). Search was limited to papers involving human subjects and published in the English language between 01/01/2000 to $31 / 03 / 2020$. The original search was created in PubMed and adjusted thereafter to the other databases.

\section{Eligibility criteria}

Randomized controlled clinical studies, cross-sectional, cohort and case-control studies reporting data on COPD patients with periodontal disease and its treatment were considered eligible for inclusion. Both prospective and retrospective studies were included and assessed separately. Case reports, case series, and systematic reviews were excluded.

\section{Definitions, interventions and outcomes measures}

COPD diagnosis was based on Global Initiative for Chronic Obstructive Lung Disease report[1], that is a post bronchodilator ratio of forced expiratory volume in 1st second $\left(\mathrm{FEV}_{1}\right)$ to forced vital capacity (FVC) $\left(\mathrm{FEV}_{1} / \mathrm{FVC}<70 \%\right)[1]$ or on previous track records and physicians' reports. For the diagnosis of periodontitis, we used the criteria employed by the authors of the individual papers as long as they were validated.

Interventions included non-surgical or surgical periodontal therapy, which was established by hand instruments or ultrasonic devices. The effectiveness of periodontal treatment was assessed according to the results of before-after periodontal treatment studies and from reports which compared COPD patients who received periodontal treatment against those who received no treatment.

The primary outcome measures included the effect of non-surgical, surgical and supportive periodontal therapy on the frequency of COPD exacerbations, lung function $\left(\mathrm{FEV}_{1}, \mathrm{FEV}_{1} / \mathrm{FVC}\right)$ and quality of life. The 
Table 1 Search strategy used for each database with the corresponding results

\begin{tabular}{|c|c|c|c|}
\hline Electronic databases & Search strategy & Limits & Hits \\
\hline Pubmed & $\begin{array}{l}\text { (Periodontitis OR periodontal disease OR gum disease OR periodontal } \\
\text { health OR periodontal therapy OR periodontal treatment OR root scaling } \\
\text { OR periodontal debridement OR root planing OR oral hygiene) AND } \\
\text { (chronic obstructive pulmonary disease OR chronic bronchitis OR emphy- } \\
\text { sema OR COPD) }\end{array}$ & $\begin{array}{l}\text { Publication Date 01/01/2000-31/03/2020 } \\
\text { English language } \\
\text { Human species }\end{array}$ & 185 \\
\hline Scopus & $\begin{array}{l}\text { (Periodontitis OR periodontal disease OR gum disease OR periodontal } \\
\text { health OR periodontal therapy OR periodontal treatment OR root scaling } \\
\text { OR periodontal debridement OR root planing OR oral hygiene) AND } \\
\text { (chronic obstructive pulmonary disease OR chronic bronchitis OR emphy- } \\
\text { sema OR COPD) }\end{array}$ & $\begin{array}{l}\text { Year 2000-2020 } \\
\text { English language }\end{array}$ & 55 \\
\hline Virtual Health Library & $\begin{array}{l}\text { (Periodontitis OR periodontal disease OR gum disease OR periodontal } \\
\text { health OR periodontal therapy OR periodontal treatment OR root scaling } \\
\text { OR periodontal debridement OR root planing OR oral hygiene) AND } \\
\text { (chronic obstructive pulmonary disease OR chronic bronchitis OR emphy- } \\
\text { sema OR COPD) }\end{array}$ & $\begin{array}{l}\text { Year 2000-2020 } \\
\text { English Language }\end{array}$ & 252 \\
\hline ScienceDirect & $\begin{array}{l}\text { ("Periodontitis" OR "periodontal disease" OR "periodontal therapy" OR "peri- } \\
\text { odontal treatment" OR "root scaling" OR "periodontal debridement" OR } \\
\text { "root planing" OR "oral hygiene") AND ("chronic obstructive pulmonary } \\
\text { disease") }\end{array}$ & $\begin{array}{l}\text { Year 2000-2020 } \\
\text { Article types Research articles }\end{array}$ & 386 \\
\hline Wiley Online Library & $\begin{array}{l}\text { (Periodontitis OR periodontal disease OR gum disease OR periodontal } \\
\text { health OR periodontal therapy OR periodontal treatment OR root scaling } \\
\text { OR periodontal debridement OR root planing OR oral hygiene) AND } \\
\text { (chronic obstructive pulmonary disease OR chronic bronchitis OR emphy- } \\
\text { sema OR COPD) }\end{array}$ & $\begin{array}{l}\text { Publication date } 01 / 2000-03 / 2020 \\
\text { Publication type Journals }\end{array}$ & 213 \\
\hline Web of Science & $\begin{array}{l}\text { (Periodontitis OR periodontal disease OR gum disease OR periodontal } \\
\text { health OR periodontal therapy OR periodontal treatment OR root scaling } \\
\text { OR periodontal debridement OR root planing OR oral hygiene) AND } \\
\text { (chronic obstructive pulmonary disease OR chronic bronchitis OR emphy- } \\
\text { sema OR COPD) }\end{array}$ & $\begin{array}{l}\text { Publication Years 2000-2020 } \\
\text { Document Types Article } \\
\text { Language English }\end{array}$ & 161 \\
\hline $\begin{array}{l}\text { Proquest Dissertation } \\
\text { and Theses Global }\end{array}$ & $\begin{array}{l}\text { (Periodontitis OR periodontal disease OR gum disease OR periodontal } \\
\text { health OR periodontal therapy OR periodontal treatment OR root scaling } \\
\text { OR periodontal debridement OR root planing OR oral hygiene) AND } \\
\text { (chronic obstructive pulmonary disease OR chronic bronchitis OR emphy- } \\
\text { sema OR COPD) }\end{array}$ & $\begin{array}{l}\text { Publication Date } 01 / 01 / 2020-31 / 03 / 20 \\
\text { Limit to Title and Abstract } \\
\text { English Language }\end{array}$ & 124 \\
\hline Google Scholar & $\begin{array}{l}\text { ("Periodontitis" OR "periodontal disease" OR "periodontal health" OR } \\
\text { "periodontal therapy" OR "periodontal treatment" OR "root scaling" OR } \\
\text { "root planing" OR "oral hygiene") AND ("COPD" OR "chronic obstructive } \\
\text { pulmonary disease") }\end{array}$ & $\begin{array}{l}\text { Limit to Title } \\
\text { English language } \\
\text { Publication Date } 2000-2020\end{array}$ & 66 \\
\hline
\end{tabular}

secondary outcome measures included: oral hygiene and periodontal indices, radiographic alveolar bone loss, levels of inflammatory biomarkers, periodontal bacterial burden, comorbidities, hospitalizations and mortality.

\section{Data management, study selection and data extraction}

Literature search results were uploaded to Distiller Systematic Review (DSR) software, an Internet based software program that facilitates collaboration among reviewers during the study selection process. After duplicates' removal, the team developed screening questions and forms for level 1 and 2 assessments based on the eligibility criteria. Citation abstracts and screening questions were uploaded to DSR.

Studies were screened by two independent reviewers (IA, AV) who assessed the titles and abstracts of each article. Disagreements were resolved by discussion, but if decision was inconclusive, the assessment of the contentious study continued to full text screening level. At that stage, the same authors carried out the independent appraisal of each article, reasons for exclusion were recorded and in case of disagreement a third author adjudicated unresolved quarrel.

Data were then extracted from each article using a custom data extraction sheet and included the following: principal author, publication year, country, study design, study population, periodontal variables, periodontal therapy techniques, COPD assessment methods and main results.

\section{Risk of bias in individual studies}

The risk of bias of randomized clinical trials was assessed with Cochrane risk of bias tool (RoB2), which assesses 
the following five domains: bias arising from randomization process, bias due to deviation from intervention, bias due to missing outcome data, bias in measurement of the outcome and bias in selection of reported results. Possible risk of bias judgments were "low risk of bias", "some concerns" and "high risk of bias" [19].

Risk of bias assessment was performed independently by two review authors (IA and AV) and any disagreement was resolved by discussion. The risk of bias of nonrandomized studies was assessed with Cochrane risk of bias tool for non-randomized studies of intervention (ROBINS-I tool) which assesses the following 7 domains of bias: (1) confounding (2) selection of participants into the study (3) classification of interventions (4) deviations from intended interventions (5) missing data (6) measurement of outcomes and (7) selection of the reported result. Possible risk of bias judgments were: "low risk of bias", "moderate risk of bias", "serious risk of bias", "critical risk of bias" and "no information" [20].

\section{Risk of bias across studies}

If a sufficient number of trials were identified $(n \geq 10)$, analyses were planned to identify reporting biases ("small-study effects" and/or "publication bias"), through the inspection of a contour-enhanced funnel plot and through respective statistical tests for funnel plot asymmetry and identification of missing studies [21].

The overall quality of evidence (confidence in effect estimates) was judged using the Grading of Recommendations Assessment, Development and Evaluation (GRADE) working group methodology [22], according to a recent guidance on combining randomized with nonrandomized studies [23]. A table with a summary of findings was constructed using the format by Carrasco-Labra et al. [24] GRADEpro software [25] was used to assess the quality of evidence across the domains of risk of bias, consistency, directness, precision and publication bias. Quality was adjudicated as "high" (further research is very unlikely to change our confidence in the effect estimate), "moderate" (further research is likely to have an important impact on our confidence in the effect estimate and may change the estimate), "low" (further research is very likely to have an important impact on our confidence in the effect estimate and is likely to change the estimate) and "very low" (very uncertain about the estimate effect).

\section{Summary measures and synthesis of results}

Data were summarized and considered suitable for pooling if similar groups were reported (or could be formed) and the same outcomes were reported. For dichotomous outcomes risk ratios (RR) with 95\% confidence intervals (CIs) would be computed. For continuous outcomes weighted mean differences (95\% CIs) or standardized mean differences (95\% CIs) would be analyzed. A random-effects model as proposed by DerSimonian and Laird was chosen a priori as the primary method to assess all pooled estimates, as the observed treatment effect was expected to differ across studies due to differences in samples and implementations [26].

The extent and impact of between-study heterogeneity would be assessed by inspecting the forest plots for the localization of heterogeneity, by calculating the $\mathrm{I}^{2}$ statistic and by the magnitude and direction of effects. The $95 \%$ CIs around $\mathrm{I}^{2}$ would be calculated according to the noncentral $\mathrm{x}^{2}$ approximation of $\mathrm{Q}$. In case of considerable unexplained heterogeneity $\left(\mathrm{I}^{2}>75 \%\right)$ data would be analyzed, but not pooled. If heterogeneity was substantial, a meta-analysis would not be performed, but a systematic narrative synthesis would be provided, presenting data in text and tables to summarize and explain the characteristics and findings of the included studies.

\section{Results}

Study selection and characteristics

A flow diagram (Fig. 1) depicts the process that we employed in order to identify relevant articles. Out of 1442 initially retrieved studies, 7 studies were considered eligible for inclusion in this systematic review. Table 2 demonstrates the major characteristics of the 7 studies. Three of the included studies were RCTs [27-29], 2 were case-control studies [30, 31], 1 was cross-sectional study [32] and 1 was a before-after treatment cohort study [33]. These studies were conducted in India [27, 33], Taiwan [30], China [29, 32], Turkey [31] and USA [28] and took place at university and hospital settings. Due to both small sample sizes from most included studies, but most importantly due to differences in the study design, types of interventions and comparisons as well as their methods of reporting results and because of the low quality of the eligible articles, a meta-analysis could not be performed. Hence, the respective results of each study are presented without being statistically processed.

The diagnosis of COPD was based on GOLD criteria [27, 29, 31-33], medical records [28] and ICD-9 classification [30]. The diagnosis of COPD exacerbations was based on the GOLD criteria [29, 32], ICD-9 classification [30] and symptom worsening requiring treatment adjustment and/or hospitalization [31]. COPD exacerbations were reported by the patients [32], by the investigators [29] or were reported by the patient and confirmed by the investigator [31]; while 2 studies have also provided data on COPD exacerbations prior to periodontal interventions based on patients' recall $[29,31]$.

The diagnosis of periodontitis was based on a mean attachment loss (MAL) $\geq 1.5 \mathrm{~mm}[28]$, a community 


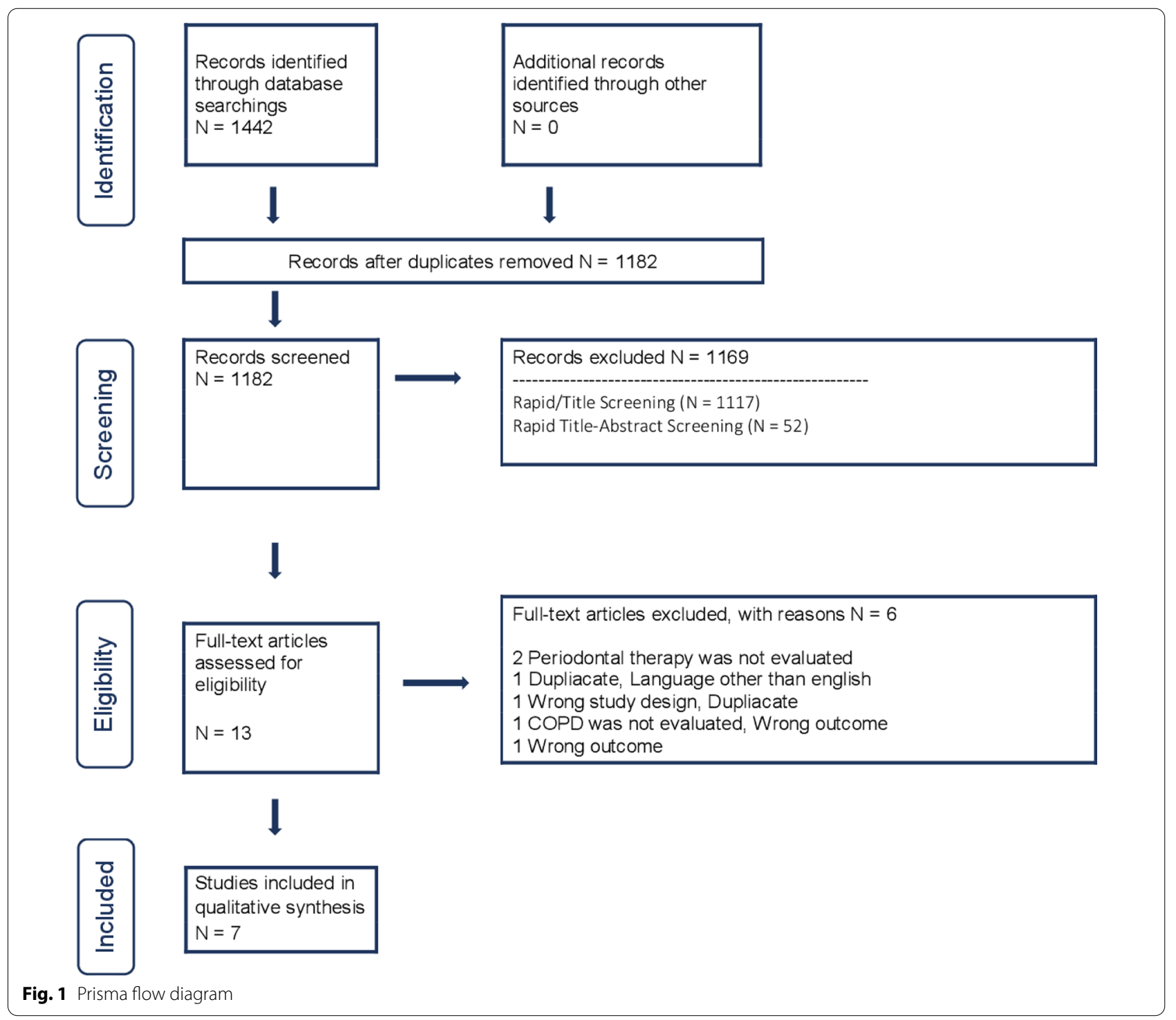

periodontal index $(\mathrm{CPI})>3$ and a loss of attachment $(\mathrm{LOA})>1$ [33], the presence of at least one site with a pocket probing depth (PPD) $>3 \mathrm{~mm}$ and periodontal clinical attachment loss (CAL) $>3 \mathrm{~mm}[29]$, the ICD-9 classification[30], the American Academy of Periodontology Workshop 1999 guidelines [31, 34] and on oral/periodontal health indices [27, 32]. Quality of life was assessed with the Saint George Respiratory Questionnaire (SGRQ) in 2 studies $[27,28]$. The effect of periodontal treatment on lung function variables was investigated in 2 other studies $[29,33]$.

Periodontal therapy was established through scaling and root planning using hand instruments [27], supragingival scaling, hygiene instructions and preprocedural antibiotics [33], subgingival curettage, root planning and periodontal flap surgery (if needed) [30], oral hygiene instructions, supra- and subgingival scaling using hand instruments and ultrasonic devices [28, $29,31]$. In another study, information about the frequency of previous supragingival scaling procedures was obtained by interview [32].

\section{Risk of bias within studies}

Risk of bias for RCTs was judged as "some concerns" in one trial[28] and "high" in two trials [27, 29]. With regard to the rest of the studies, serious methodological limitations were found for two studies [32,33] and moderate for another two [30,31]. Risk of bias summary plots are depicted in Figs. 2 and 3. 


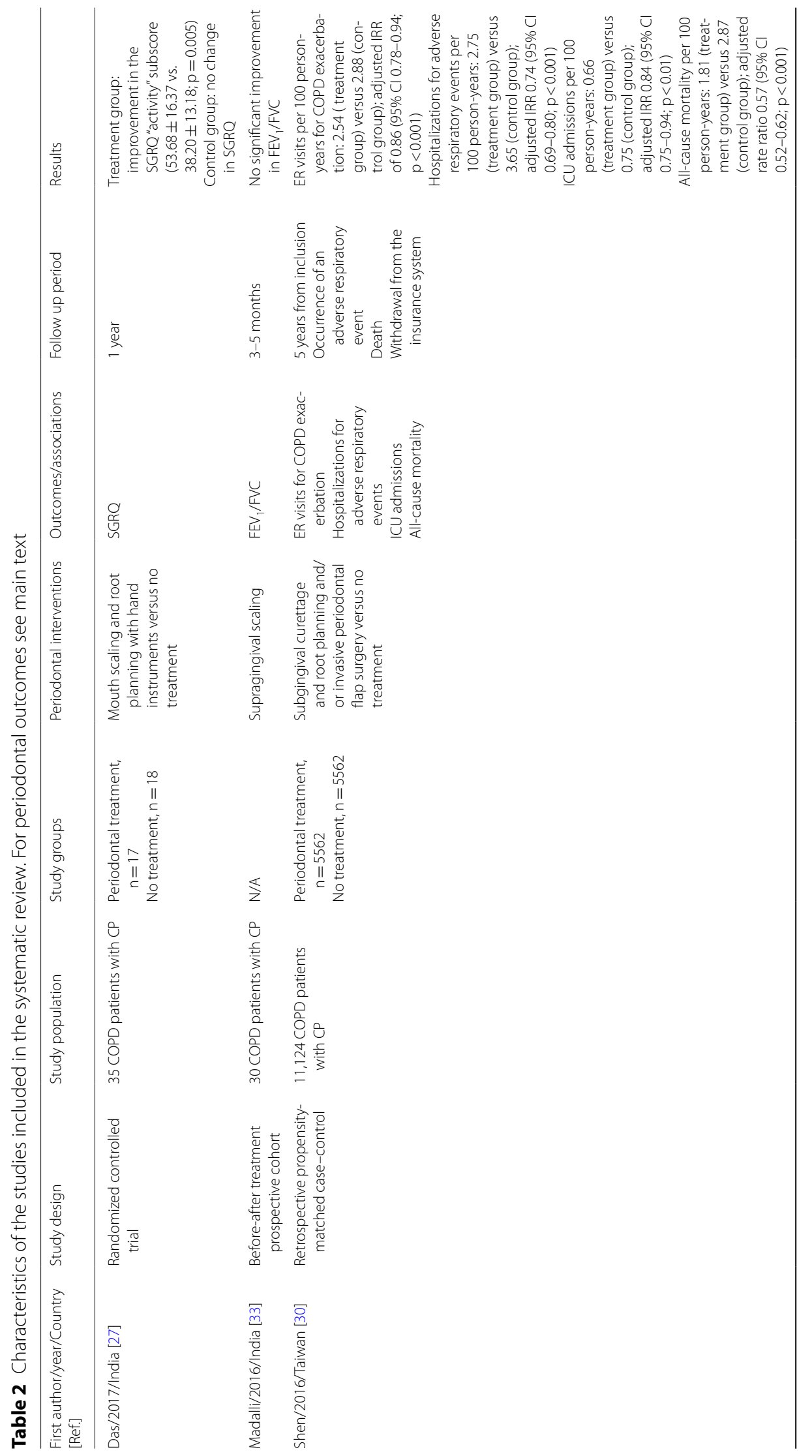


Apessos et al. BMC Pulm Med $\quad$ (2021) 21:92

Page 7 of 16

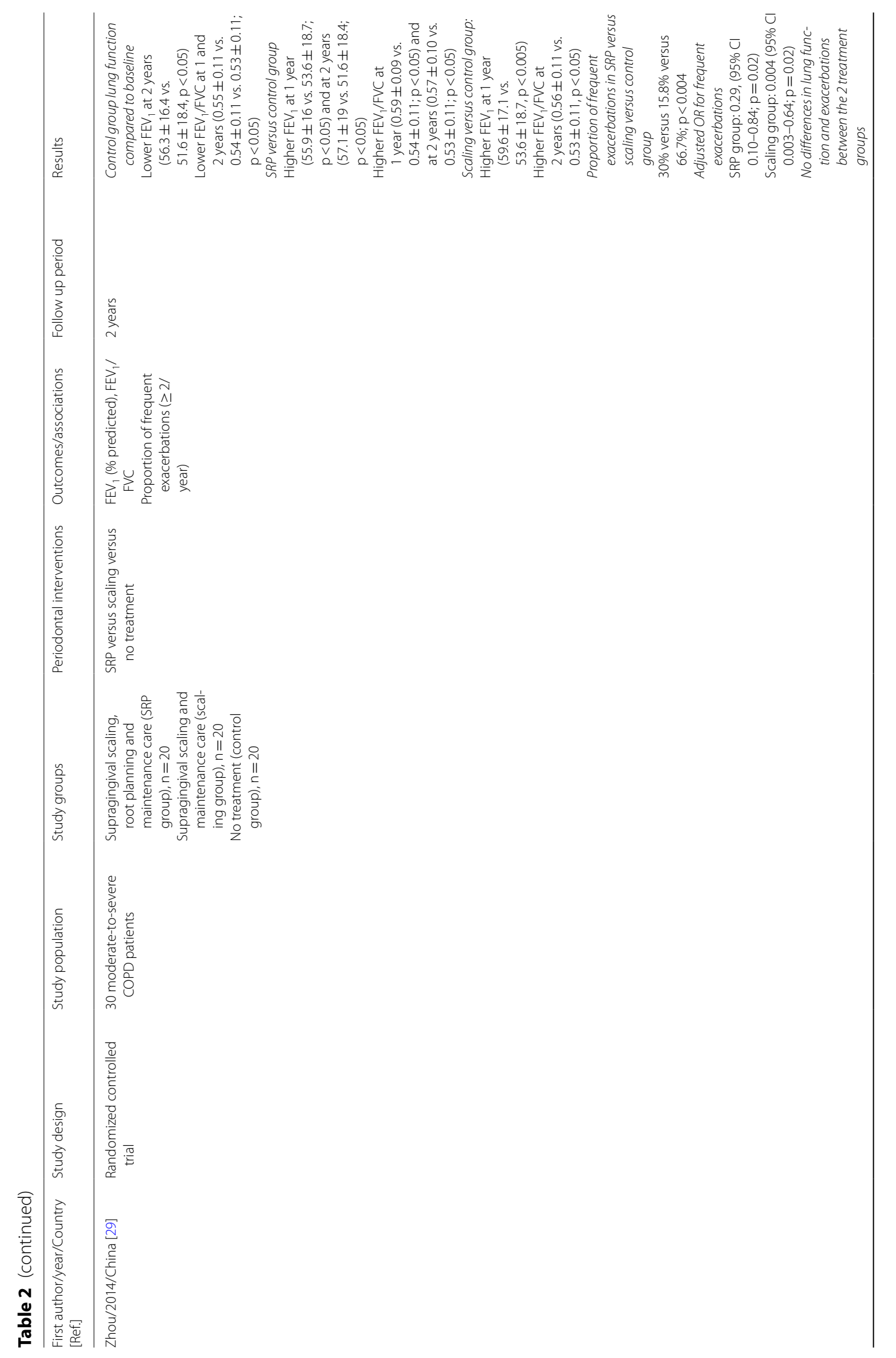


Apessos et al. BMC Pulm Med $\quad$ (2021) 21:92

Page 8 of 16

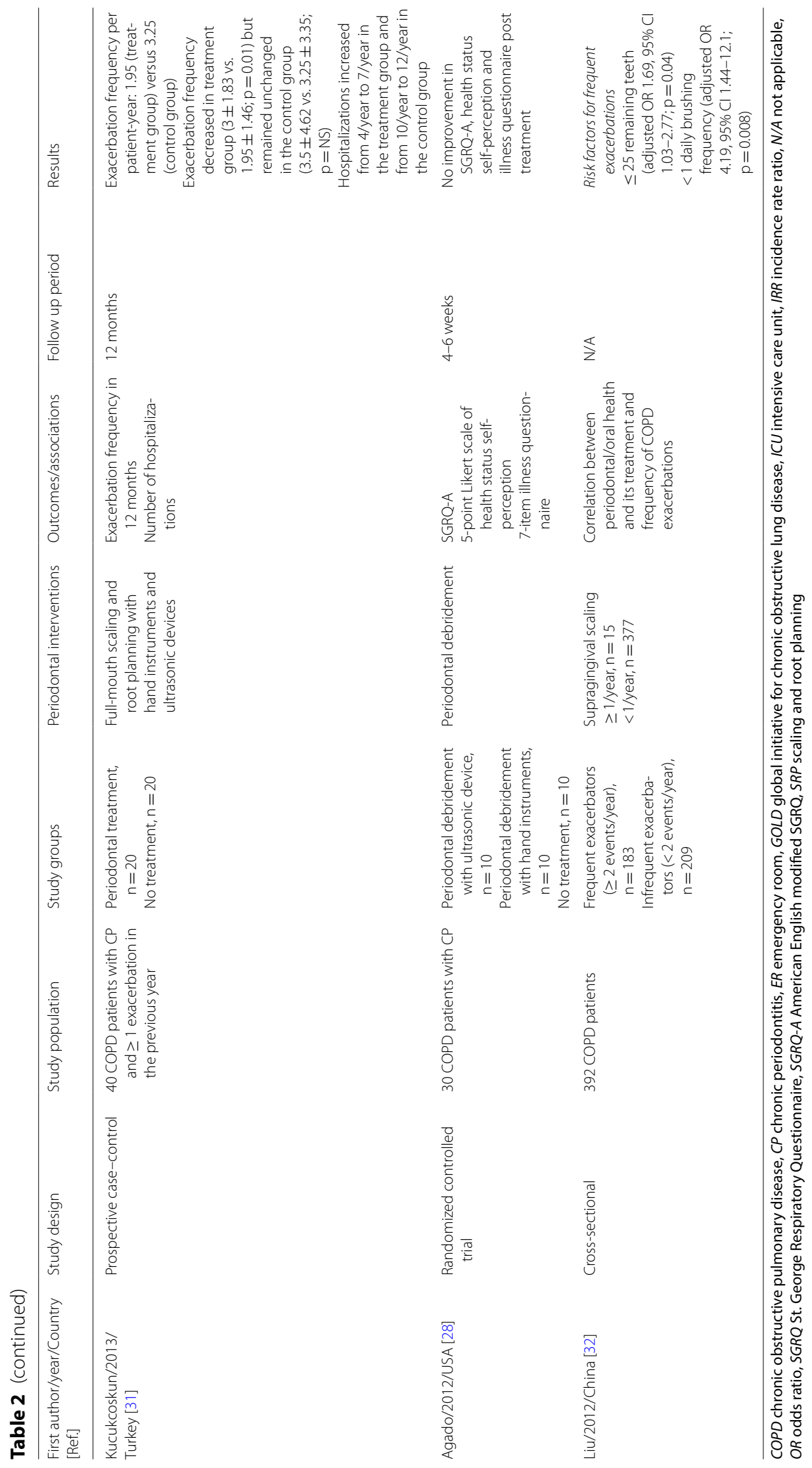




\begin{tabular}{|l} 
Das et al, 2017, India \\
Agado et al, 2012, USA \\
Fig. 2 Risk of bias assessment for randomized trials (RoB2) \\
D5: Bias in selection of the reported result. \\
Domains: \\
D2: Bias arising from the randomization process \\
D3: Bias due to deviations from intended intervention. \\
D4: Bias in meansing outcome data.
\end{tabular}

Madalli et al, 2016, India
Kucukcoskun et al, 2013, Turkey
Fig. 3 Risk of bias assessment for non-randomized studies (ROBINS-I)

\section{Results of data synthesis Quality of life}

Two studies [27, 28] evaluated the effect of periodontal therapy on quality of life. Das et al. [28] randomized 35 COPD patients (diagnosed according to the GOLD spirometric criteria) to receive either periodontal treatment (treatment group, $\mathrm{n}=18$ ) or no treatment (control group, $\mathrm{n}=17$ ). Interventions included full-mouth scaling and root planning with hand instruments as well as oral hygiene instructions, while the effect of periodontal therapy on quality of life was assessed with the SGRQ score. All patients were reassessed at 1-year post-treatment for significant changes in periodontal and oral parameters and in SGRQ scores. With respect to the intervention group in this study significant improvements were observed in the Oral Hygiene Index-Simplified (OHIS), Gingival Index (GI) and CAL, but not in PPD, while no differences in any of the appraised parameters were 
noted in the control group. The SGRQ "activity" subscore significantly improved in the treatment group post treatment $(53.68 \pm 16.37$ vs. $38.20 \pm 13.18 ; \mathrm{p}=0.005)$, but no differences in any of the SGRQ subscores were observed in the control group. At the end of the 1-year follow-up period, no differences were noted in the SGRQ subscores between the two groups with the exception of the "activity" subscore which was significantly better in the intervention group ( $38.20 \pm 13.18$ vs. $56.48 \pm 18.31 ; \mathrm{p}<0.01)$.

Agado et al. [28] randomized 30 COPD patients (recruited from medical databases) with chronic periodontitis (MAL $\geq 1.5 \mathrm{~mm}$ ) into periodontal therapy with ultrasonic instrumentation $(n=10)$, periodontal therapy with hand instruments $(n=10)$ or no treatment $(n=10)$ for a follow-up period of 4-6 weeks; periodontal treatment was completed when all clinically detectable deposits had been removed. The effect of periodontal therapy on quality of life was evaluated with the American English modified SGRQ score (SGRQ-A), while patients graded their perception of health status on a 5-point Likert scale from "very poor" to "very good". In addition, all participants provided a yes/no response to a customary made 7-item "illness questionnaire" which interrogated respiratory or other illness within the previous 4 weeks, physician visits, antibiotic use, use of respiratory medications, respiratory problems after dental care and avoidance of dental care due to respiratory ailments. All questionnaires were issued by an investigator blinded to the type of intervention. At the end of the follow-up period no significant difference was noted between the 3 groups in SGRQ-A total scores, self-assessment of health scores and "illness questionnaire" and no improvement between pre- and post-test. However, each group showed a significant improvement in the SGRQ-A "activity" subscore but with no interactions between groups.

\section{Lung function}

Two studies [29, 33] evaluated the effect of periodontal therapy on lung function. Madalli et al. ${ }^{29}$ conducted a before-after treatment prospective cohort study of 30 COPD patients with periodontal disease to investigate the effect of periodontal treatment on $\mathrm{FEV}_{1} / \mathrm{FVC}$ variables. COPD diagnosis was based on the GOLD spirometric criteria and the diagnostic criteria for periodontal disease included a CPI score $\geq 3$ and a LOA score $\geq 1$. Interventions included supragingival scaling and oral hygiene instructions and all patients were re-assessed after 3-5 months. This study observed no significant changes in CPI and LOA scores, while no significant improvement in $\mathrm{FEV}_{1} / \mathrm{FVC}$ was also noted $(48.08 \pm 12.01$ vs. 51.25 $\pm 12.29 ; \mathrm{p}=\mathrm{NS}$ ). Zhou et al. [29] randomized 60 moderate-to-severe COPD patients (diagnosed according to the GOLD criteria) into scaling, root planning and maintenance care (SRP group, $n=20$ ), supragingival scaling alone and maintenance supragingival scaling (scaling group, $\mathrm{n}=20$ ) or no treatment (control group, $\mathrm{n}=20$ ). Periodontal disease was considered based on the presence of at least one site with a PPD $>3 \mathrm{~mm}$ and a CAL $>3 \mathrm{~mm}$. All patients were re-assessed for changes in periodontal variables, namely $\mathrm{PPD}, \mathrm{CAL}$, bleeding index (BI) and plaque index (PI) at 6 months, 1 year and 2 years, while lung function variables $\left(\mathrm{FEV}_{1}, \mathrm{FEV}_{1} / \mathrm{FVC}\right)$ were re-evaluated at 1 year and 2 years. This trial observed significant improvements in both treatment groups at 6 months, 1 year and 2 years for all the appraised periodontal parameters, whereas no improvements were noted in the control group; all periodontal variables were worse in the control group as compared to each of the treatment groups at any of the investigated time points. With respect to the lung function variables, when compared to baseline, the control group exhibited a significant decrease in $\mathrm{FEV}_{1}$ (\% predicted) at 2 years $(56.3 \pm 16.4$ vs. $51.6 \pm 18.4, \mathrm{p}<0.05)$ and in $\mathrm{FEV}_{1} / \mathrm{FVC}$ at 1 and 2 years $(0.55 \pm 0.11$ vs. $0.54 \pm 0.11$ vs. $0.53 \pm 0.11$, respectively; $\mathrm{p}<0.05)$. When compared to the control group, the SRP group showed significantly improved levels of $\mathrm{FEV}_{1}$ (\% predicted) and $\mathrm{FEV}_{1} / \mathrm{FVC}$ at 1 year $(55.9 \pm 16$ vs. $53.6 \pm 18.7$ and $0.59 \pm 0.09$ vs. $0.54 \pm 0.11$, respectively; $\mathrm{p}<0.05)$ and at 2 years $(57.1 \pm 19$ vs. $51.6 \pm 18.4$ and $0.57 \pm 0.10$ vs. $0.53 \pm 0.11$, respectively; $\mathrm{p}<0.05$ ). Likewise, when the scaling group was compared to controls, it exhibited a significantly higher $\mathrm{FEV}_{1}$ (\% predicted) at 1 year $(59.6 \pm 17.1$ vs. $53.6 \pm 18.7, \mathrm{p}<0.005)$ and $\mathrm{FEV}_{1} /$ FVC at 2 years $(0.56 \pm 0.11$ vs. $0.53 \pm 0.11, \mathrm{p}<0.05)$. No differences in lung function were observed between the two treatment groups at any of the abovementioned time points.

\section{COPD exacerbations}

Four studies [29-32] evaluated the effect of periodontal therapy on the frequency of COPD exacerbations. In the previously mentioned RCT by Zhou et al., COPD exacerbations post-randomization were defined according to the GOLD criteria and their frequencies were assessed at 6 months, 1 year and 2 years, while their frequencies during the last year prior to randomization were self-reported by the patients; patients with $\geq 2$ events / year were defined as "frequent" exacerbators. At 2 years, the SPR and the scaling groups showed a lower proportion of "frequent" exacerbations as compared to controls ( $30 \%$ vs. $15.8 \%$ vs. $66.7 \%$, respectively; $\mathrm{p}<0.004)$. In a multivariate logistic regression model, the calculated OR for "frequent" exacerbations was 0.29 (95\% CI $0.10-0.84 ; \mathrm{p}=0.02)$ for the SRP group and 0.004 (95\% CI $0.003-0.64 ; \mathrm{p}=0.02$ ) for the scaling group after adjusting for age, gender, body mass index (BMI) and baseline 
frequency of exacerbations. No differences were noted in the frequency of COPD exacerbations between the two treatment groups.

Shen et al. [30] conducted a population-based, retrospective case-control study based on the Taiwan National Health Insurance claims data. COPD and periodontal disease diagnosis, periodontal interventions and adverse respiratory effects were identified based on the database's ICD-9 classification. The study population consisted of 5562 COPD individuals that received periodontal therapy and 5562 propensity-matched controls. All subjects were followed up to a maximum of 5 years or until death, occurrence of an adverse respiratory event or withdrawal from the insurance system. Periodontal interventions included supragingival scaling and root planning as well as periodontal flap surgery, depending on the severity of the disease. The primary outcomes included emergency room (ER) visit and hospitalization rates following acute COPD exacerbation, pneumonia or acute respiratory failure. Overall, all types of adverse respiratory events were less frequent in the treatment group as opposed to the control group. After adjusting for confounders, including age, gender, residential area, occupation, income and comorbidities, the ER visit rate per 100 person-years for COPD exacerbation was 2.54 for the treatment group versus 2.88 for the control group, for an adjusted incidence rate ratio (IRR) of 0.86 (95\% CI 0.78-0.94; $\mathrm{p}<0.001)$.

Kucukcoskun et al. [31] conducted a prospective casecontrol study consisting of 40 patients with COPD, diagnosed according to the GOLD criteria with a history of $\geq 1$ exacerbation during the previous year and with moderate-to-severe chronic periodontitis based on the 1999 American Academy of Periodontology Workshop guidelines. COPD exacerbation was defined as a worsening in the baseline respiratory symptoms lasting at least 2 days and necessitating oral steroids and antibiotics (moderate exacerbation) or hospitalization (severe exacerbation). Patients were allotted to periodontal treatment (oral hygiene, full mouth scaling and root planning with hand instruments and ultrasonic devices completed over 3 visits, $\mathrm{n}=20)$ or no treatment $(\mathrm{n}=20)$ according to their access to periodontal services. Exacerbations were self-reported by the patients and confirmed by the investigator, while the number of exacerbations and hospitalizations during the last year before enrollment was also registered. Periodontal procedures were performed by a dentist blind to the study design and COPD severity and all patients were re-evaluated at 6 and 12 months. With respect to the periodontal parameters, when compared to baseline, the treatment group exhibited significant improvements in BP, GI, PPD and CAL at 6 months; at 12 months, this improvement remained significant only for CAL. On the other hand, in the control group most of the periodontal parameters showed significant deterioration at 6 (GI, PPD, PI and CAL) and 12 months (GI, PPD, and $\mathrm{CAL}$ ). The frequency of exacerbations per patientyear was 1.95 events in the treatment group versus 3.25 events in the control group $(\mathrm{p}=0.01)$. After 1 year of follow-up, exacerbation frequency, was significantly reduced in the treatment group $(3 \pm 1.83$ vs. $1.95 \pm 1.46$; $\mathrm{p}=0.01)$ but remained unchanged in the control group $(3.5 \pm 4.62$ vs. $3.25 \pm 3.35 ; \mathrm{p}=\mathrm{NS})$.

Liu et al. [32] conducted a cross-sectional study investigating the role of periodontal health on the frequency of COPD exacerbations. The diagnosis and definition of COPD exacerbation were based on GOLD criteria. Overall, 392 COPD patient were assessed for the presence and severity of periodontal disease and were interviewed about oral health habits, history of dental visits and frequency of supragingival scaling procedures $(\geq 1$ time/year vs. $<1$ time/year) during the last 12 months. Periodontal examination was conducted by an investigator blinded to COPD severity. Patients also provided information about the frequency of exacerbations during the previous 12 months with patients with $<2$ events classified as "infrequent" exacerbators $(n=209)$, whereas those with $\geq 2$ events considered "frequent" exacerbators $(\mathrm{n}=183)$. In a multivariate logistic regression model adjusted for age, gender, BMI and smoking, $\leq 25$ remaining teeth (adjusted OR 1.63, 95\% CI 1.01-2.61; $\mathrm{p}=0.045$ ), a $\mathrm{PI}>2$ (adjusted OR 1.93, 95\% CI 1.09-3.42; $\mathrm{p}=0.02$ ) and $<1$ /day brushing frequency (adjusted OR 4.5, 95\% CI $1.6-12.7 ; \mathrm{p}=0.004)$ were the independent predictors of "frequent" exacerbations. When COPD severity and dyspnea scores were additionally considered in the model, this association was still significant only for fewer remaining teeth (adjusted OR 1.69, 95\% CI 1.03-2.77; $\mathrm{p}=0.04$ ) and lower daily brushing frequency (adjusted OR 4.19, 95\% CI 1.44-12.1; $\mathrm{p}=0.008$ ). The frequency of supragingival treatment was not included in any of the models, however the number of patients who underwent frequent treatment ( $\geq 1$ time/year) was very low (4\%).

\section{Secondary outcomes \\ Periodontal outcomes}

Regarding the periodontal outcomes, the appraised parameters presented amelioration or no change. In the RCT by Das et al., periodontal therapy with full-mouth scaling and root planning with hand instruments combined with oral hygiene was associated with improvements in OHI-S ( $3.38 \pm 1.27$ vs. $2.32 \pm 1.21 ; \mathrm{p}=0.018)$, GI $(1.79 \pm 0.54$ vs. $1.19 \pm 0.47 ; \mathrm{p}=0.001)$ and $\mathrm{CAL}$ $(4.17 \pm 0.75$ vs. $3.26 \pm 0.90 ; \mathrm{p}=0.003)$ at 1 year post-treatment [27]. In the case-control study by Kucukcoskun et al. full mouth scaling and root planning with hand 
instruments plus oral hygiene effected improvements in BOP\% (58 \pm 28 vs. $47 \pm 19 ; \mathrm{p}<0.01)$, GI $(1.52 \pm 0.37$ vs. $1.34 \pm 0.23 ; \mathrm{p}<0.01), \operatorname{PPD}(2.70 \pm 0.66$ vs. $2.39 \pm 0.41$; $\mathrm{p}<0.01)$ and CAL $(3.73 \pm 1.16$ vs. $3.39 \pm 1.01 ; \mathrm{p}<0.01)$ at 6 months, as compared to baseline; at 12 months CAL continued to show significant improvement with respect to its baseline value $(3.73 \pm 1.16$ vs. $3.52 \pm 1.02 ; \mathrm{p}<0.01)$ [31]. Likewise, in the RCT by Zhou et al. [29], all assessed periodontal parameters (i.e. PD, CAL, BI, PI), exhibited significant improvements for both the SRP and the scaling group at any time point ( 6 months, 1 year, 2 years). On the contrary, supragingival scaling plus oral hygiene did not result in any improvement in CPI and LOA scores $(4 \pm 0.51$ vs. $4.0 \pm 0.48$ and $2 \pm 0.37$ vs. $2 \pm 0.37$, respectively; $\mathrm{p}=\mathrm{NS}$ ) after 3-5 months as reported by Madalli and colleagues. Radiographic alveolar bone loss and levels of inflammatory biomarkers were not assessed by any of the included studies.

\section{Periodontal pathogens, hospitalizations, mortality and comorbidities.}

In the study by Madalli et al. [33] the proportion of COPD patients with a positive sputum sample for Porphyromonas gingivalis decreased from $14 / 30$ to $8 / 30$ at 3-5 months post treatment, although this finding was not significant. In the case-control study by Kucukcoskun et al. [31], 1-year hospitalization rate post-treatment increased as compared to the previous year both in the treatment (from 4 to 7 events) and in the control group (from 10 to 12 events). On the other hand, in the retrospective case-control study by Shen et al. [30] the respective hospitalization rates per 100 person-years for all types of adverse respiratory events were 2.75 for the treatment group versus 3.65 per 100 person-years for the control group (adjusted IRR 0.74, 95\% CI 0.69$0.80 ; \mathrm{p}<0.001)$. In addition, ICU admissions per 100 person-years were 0.66 for the treatment group versus 0.75 for the control group (adjusted IRR 0.84, 95\% CI $0.75-0.94 ; \mathrm{p}<0.01$ ), while the all-cause mortality rate was $37 \%$ lower for those treated for periodontal disease (adjusted rate ratio $0.57,95 \%$ CI $0.52-0.62$; $\mathrm{p}<0.001$ ). No study reported on the effects of periodontal treatment on the prevalence and severity of COPD-associated comorbidities.

\section{Risk of bias across studies}

The quality of evidence for all outcomes ranged from moderate to very low (Table 3). The main reason for downgrading the quality of evidence pertained to the inclusion of non-randomized studies with serious methodological issues that most probably introduce bias and to the imprecision of estimates due to narrative synthesis. This means that further research with well-designed studies is very likely to have an important impact, and is likely to change our current estimates of effect.

\section{Discussion \\ Summary of evidence}

The present systematic review addresses the effects of non-surgical or surgical periodontal treatment on various clinical outcomes of patients suffering from COPD and periodontal disease. To the best of our knowledge this is the first study to systematically assess the direct effect of periodontal therapy on the outcomes of patients with COPD. In summary, the accumulated evidence suggests that effective periodontal treatment in patients with COPD and periodontitis is associated with a reduced frequency of COPD exacerbations and slower lung function decline, although it is unclear whether it can also improve the quality of life. In addition, based on the results of a large population-based retrospective case-control study, periodontal treatment reduces the rate of hospitalizations for respiratory events (including COPD exacerbations) and is associated with lower all-cause mortality.

COPD is associated with systemic inflammation [35] and oxidative stress [36] and is now considered a systemic disease with frequent manifestations "outside the lungs" [37]. Thus, several comorbidities including cardiometabolic ones, lung cancer, osteoporosis, anxiety and depression, obstructive sleep apnea and gastroesophageal reflux occur more frequently in COPD patients compared to the general population [38]. Considering these findings, there is an ongoing need to further identify relevant comorbidities which share common pathogenetic factors with COPD and thus could exert a negative effect on its clinical outcomes. A growing body of studies over the last years has established an association between periodontitis and COPD [11, 15-17]. Both diseases are characterized by neutrophilic inflammation with subsequent proteolytic destruction of connective tissue and share common risk factors such as age, smoking and social deprivation [9]. Accumulating evidence has identified several putative pathophysiologic mechanisms linking the two disorders including aspiration of cytokines, bacteria and neutrophils $[9,10]$, hematogenous dissemination of periodontal pathogens via gingival microulcerations [39] and hyper-reactivity of peripheral blood neutrophils to bacterial stimuli and reactive oxygen species [40]. Among these, the most compelling evidence supports the hypothesis of neutrophilic inflammation and neutrophil-mediated tissue damage $[9,10]$. Although a direct causal link cannot be unequivocally suggested based on current evidence, some studies have shown a higher loss of lung function in patients with more severe periodontal disease ${ }^{34}$. Interestingly, COPD patients receiving corticosteroids $(\mathrm{CS})$ are exposed at higher risk 


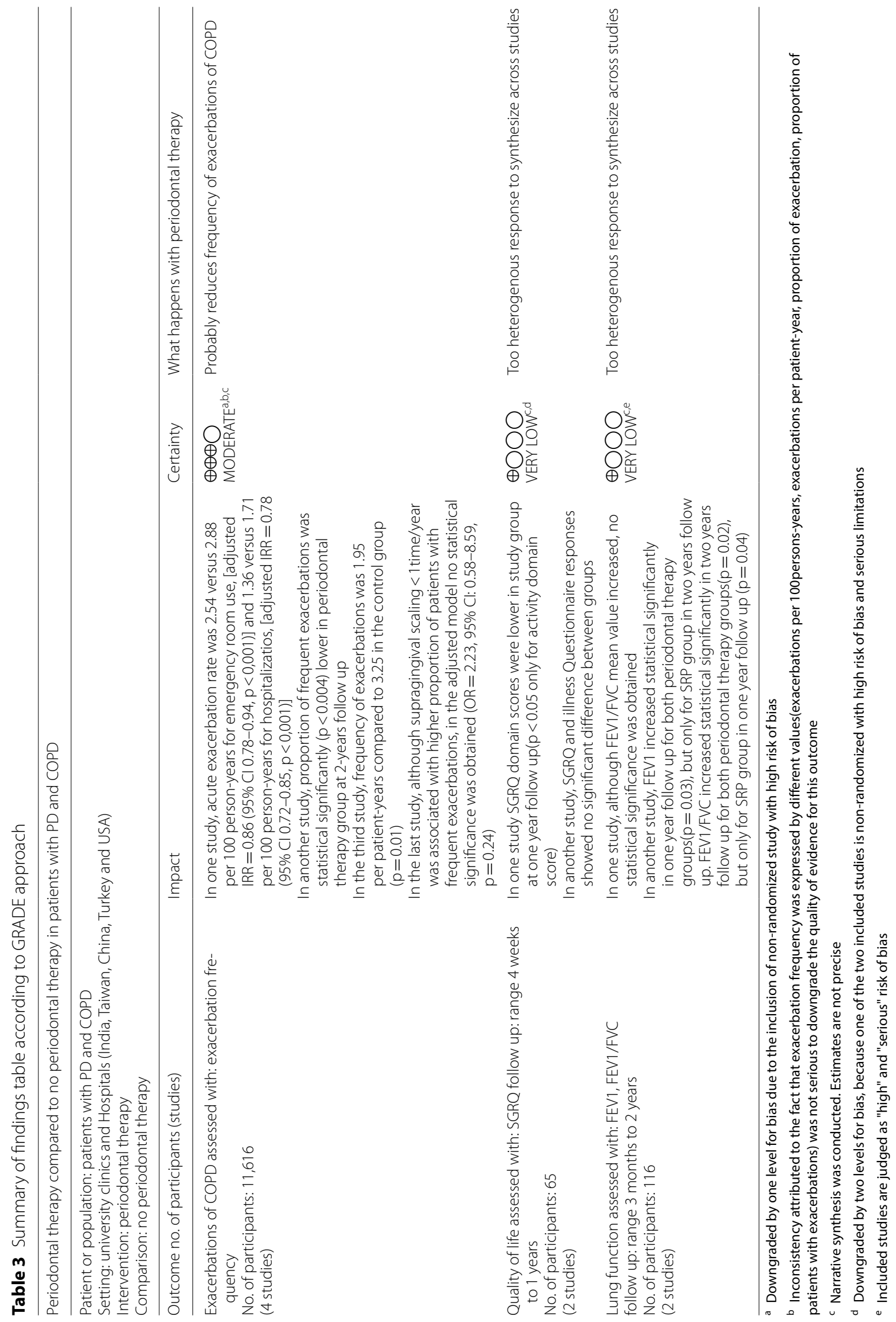


for periodontal disease as compared with those who are not; an additional issue to consider regarding the puzzling associations between COPD and periodontitis and specifically the additive effects of COPD treatment on onset and progression of periodontal disease $[41,42]$.

Several mechanisms have been proposed regarding the beneficial effects of periodontal treatment on COPD outcomes. First, periodontal therapy has been shown to reduce the concentration of various inflammatory biomarkers, potentially leading to less severe inflammatory response in patients with COPD [43, 44]. Second, removal of dental plaque may lead to a reduction of oral mucosa colonization with pathogens which are commonly isolated in respiratory and dental specimens of patients with COPD, especially during exacerbations $[45,46]$. On the other hand, some authors have reported a deterioration of respiratory symptoms due to bacterial contamination caused by aerosols produced during dental treatment with ultrasonic devices [33, 47]. Antimicrobial preprocedural mouth rinse, high volume evacuation suction and air polishing avoidance have been proposed as precautionary measures [47].

\section{Clinical relevance}

The present systematic review underscores the need for collaboration between pulmonologists and dental practitioners in the field of COPD management. Poor oral health is one of the primary risk factors for COPD exacerbations and could stand on its own right as a potentially modifiable condition $[48,49]$. On the other hand, the presence of periodontal disease should alert dental practitioners to the possibility of coexistent COPD and prompt them to refer the patient for further evaluation [29]. Future clinical trials should aim to investigate whether COPD patients will benefit from routine oral/ dental assessment, and to provide important insights on the pathophysiological link between periodontal disease and COPD.

\section{Strengths and limitations}

This systematic review was conducted according to an a priori registered protocol and has several strengths, including a comprehensive literature search, inclusion of randomized and non-randomized studies and the application of the GRADE approach to assess the overall quality of the evidence.

However, there are also several limitations that should be acknowledged. First, our search strategy was restricted to the last 20 years. However, it was our aim to evaluate the most recent data pertaining to the impact of periodontal treatment on COPD outcomes given that the most widely accepted diagnostic criteria for periodontitis and COPD were proposed in 1999 [34] and in
2001 [50], respectively and continue to be in use. In addition, the overall quality of evidence was very low for the effect of periodontal therapy on lung function and quality of life and moderate regarding its effect on COPD exacerbation frequency. Other limitations include the differences in study design and their methods of reporting results, the lack of uniformly applied definitions for COPD and periodontitis and the moderate-to-high risk of bias in individual studies. It should also be noted that in some studies exacerbations were self-reported by patients and not objectively verified by the investigator. Importantly, neither of the studies made adjustments for COPD severity according to $\mathrm{FEV}_{1}$, nor explored the effect of periodontal treatment on respiratory symptoms, both considered powerful predictors of future exacerbations and hospitalizations [51]. Of note, exacerbations and their related risk of hospitalization are strongly associated with greater lung function decline among COPD patients [52]. Interestingly, the study by Shen et al. [30] demonstrated a beneficial role of periodontal treatment both on exacerbation rate and on $\mathrm{FEV}_{1}$ decline, a finding that should be examined more concisely in future studies. Additionally, only one study investigated the role of supportive follow-up periodontal therapy on COPD outcomes $[29,53]$. Finally, the impact of periodontal treatment on prevalence and severity of COPD comorbidities has not been investigated.

\section{Conclusions}

Very low-to-moderate quality evidence suggests that periodontal treatment is associated with slower lung function decline, reduced frequency of exacerbations and less use of healthcare resources in patients with COPD and chronic periodontitis. Based on these findings we recommend that all COPD patients should be advised to undergo regular dental examination and follow up. However, because of the limitations of the available evidence, large-scale clinical trials are warranted to further elucidate the effect of periodontal treatment on COPD outcomes.

\section{Abbreviations}

COPD: Chronic obstructive pulmonary disease; RoB2: Risk of bias 2 tool; ROBINS-I: Risk of bias for non-randomized studies of intervention; GRADE: Grading of Recommendations Assessment, Development and Evaluation; PROSPERO: International prospective register of systematic reviews; CVD: Cardiovascular disease; PICO: Participants, Interventions, Comparisons, Outcomes; PRISMA-P: Preferred Reporting Items for Systematic Reviews and Meta-Analyses protocol; FEV $V_{1}$ : Forced expiratory volume in 1st second; FVC: Forced vital capacity; DSR: Distiller systematic review software; RR: Risk ratio; Cl: Confidence interval; RCT : Randomized controlled trial; MAL: Mean attachment loss; CPI: Community periodontal index; LOA: Loss of attachment; PPD: Periodontal pocket depth; CAL: Clinical attachment loss; ICD-9: International classification of diseases 9th clinical modification; OHI-S: Oral hygiene index simplified; GI: Gingival index; BI: Bleeding index; PI: Plaque index; BMI: Body mass index; CP: Chronic 
periodontitis; ER: Emergency room; GOLD: Global initiative for chronic obstructive lung disease; ICU: Intensive care unit; IRR: Incidence rate ratio; N/A: Not applicable; OR: Odds ratio; SGRQ: St. George Respiratory Questionnaire; SGRQA: American English modified SGRQ; SRP: Scaling and root planning.

\section{Supplementary Information}

The online version contains supplementary material available at https://doi. org/10.1186/s12890-021-01429-2.

Additional file 1: TableS1. Excluded studies with reasons.

\section{Acknowledgements}

Not applicable.

\section{Authors' contributions}

IA conceived the idea, designed the study and performed databases'search. IA and AV contributed to study selection, data management, data collection, risk of bias assessment within and across studies, synthesis of results, summary of evidence and drafted manuscript, figures and tables. MA resolved arguments between IA and AV (when requested to do so), contributed to data extraction and revised explicitly initial manuscript and tables. DA and PS revised the manuscript. All authors read and approved the final version of submission.

\section{Funding}

Not applicable.

\section{Availability of data and materials}

The datasets used and/or analyzed during the current study available from the corresponding author on reasonable request.

\section{Ethics approval and consent to participate}

Not applicable.

\section{Consent for publication}

Not applicable.

\section{Competing interests}

The authors declare that they have no competing interests.

\begin{abstract}
Author details
${ }^{1}$ Department of Oral Surgery, Surgical Implantology and Oral Radiology, School of Health Sciences, School of Dentistry, Aristotle University of ThessaIoniki, 54124 Thessaloniki, Greece. ${ }^{2}$ Department of Dentistry, 424 General Military Training Hospital, Thessaloniki, Greece. ${ }^{3}$ Department of Pneumonology, Medical School, Democritus University of Thrace, Alexandroupolis, Greece. ${ }^{4}$ MSc Program in Sleep Medicine, Medical School, Democritus University of Thrace, Alexandroupolis, Greece. ${ }^{5}$ Department of Respiratory Failure, General Hospital "G. Papanikolaou", Aristotle University of Thessaloniki, Thessaloniki, Greece. ${ }^{6}$ Department of Oral Medicine/Pathology, School of Dentistry, Aristotle University of Thessaloniki, Thessaloniki, Greece.
\end{abstract}

Received: 22 December 2020 Accepted: 2 February 2021

Published online: 18 March 2021

\section{References}

1. Vogelmeier CF, Criner GJ, Martinez FJ, Anzueto A, Barnes PJ, Bourbeau J, et al. Global strategy for the diagnosis, management, and prevention of chronic obstructive lung disease 2017 report. GOLD executive summary. Am J Respir Crit Care Med. 2017;195(5):557-82.

2. Mathers $C D$, Loncar D. Projections of global mortality and burden of disease from 2002 to 2030. PLoS Med. 2006;3(11):e442.

3. Wedzicha JA, Seemungal TAR. COPD exacerbations: defining their cause and prevention. Lancet Lond Engl. 2007;370(9589):786-96.

4. Rubinsztajn R, Przybyłowski T, Maskey-Warzechowska M, Karwat K, Chazan R. Exacerbations of chronic obstructive pulmonary disease and quality of life of patients. Adv Exp Med Biol. 2016;884:69-74.
5. Smith MC, Wrobel JP. Epidemiology and clinical impact of major comorbidities in patients with COPD. Int J Chron Obstruct Pulmon Dis. 2014;9:871-88.

6. Voulgaris A, Archontogeorgis K, Steiropoulos P, Papanas N. Cardiovascular disease in patients with chronic obstructive pulmonary disease, obstructive sleep apnoea syndrome and overlap syndrome. Curr Vasc Pharmacol. 2020.

7. Papapanou PN, Sanz M, Buduneli N, Dietrich T, Feres M, Fine DH, et al. Periodontitis: consensus report of workgroup 2 of the 2017 world workshop on the classification of periodontal and peri-implant diseases and conditions. J Clin Periodontol. 2018;45(Suppl 20):S162-70.

8. Tonetti MS, Jepsen S, Jin L, Otomo-Corgel J. Impact of the global burden of periodontal diseases on health, nutrition and wellbeing of mankind: A call for global action. J Clin Periodontol. 2017:44(5):456-62.

9. Hobbins S, Chapple IL, Sapey E, Stockley RA. Is periodontitis a comorbidity of COPD or can associations be explained by shared risk factors/ behaviors? Int J Chron Obstruct Pulmon Dis. 2017;4(12):1339-49.

10. Takeuchi K, Matsumoto K, Furuta M, Fukuyama S, Takeshita T, Ogata H, et al. Periodontitis is associated with chronic obstructive pulmonary disease. J Dent Res. 2019;98(5):534-40.

11. Zeng X-T, Tu M-L, Liu D-Y, Zheng D, Zhang J, Leng W. Periodontal disease and risk of chronic obstructive pulmonary disease: a meta-analysis of observational studies. PLoS ONE. 2012;7(10):e46508.

12. Shen T-C, Chang P-Y, Lin C-L, Chen C-H, Tu C-Y, Hsia T-C, et al. Risk of periodontal diseases in patients with chronic obstructive pulmonary disease: a nationwide population-based cohort study. Medicine (Baltimore). 2015;94(46):e2047.

13. Holtfreter B, Richter S, Kocher T, Dörr M, Völzke H, Ittermann T, et al. Periodontitis is related to lung volumes and airflow limitation: a crosssectional study. Eur Respir J. 2013:42(6):1524-35.

14. Peter KP, Mute BR, Doiphode SS, Bardapurkar SJ, Borkar MS, Raje DV. Association between periodontal disease and chronic obstructive pulmonary disease: a reality or just a dogma? J Periodontol. 2013;84(12):1717-23.

15. Tan L, Wang H, Pan C, Zhao J. Periodontal health and chronic obstructive pulmonary disease stratified by smoking: a meta-analysis. Int J Clin Exp Med. 2016;9(12):23190-7.

16. Shi $Q$, Zhang B, Xing H, Yang S, Xu J, Liu H. Patients with chronic obstructive pulmonary disease suffer from worse periodontal health-evidence from a meta-analysis. Front Physiol. 2018;9:33.

17. Gomes-Filho IS, Cruz SS da, Trindade SC, Passos-Soares I de S, CarvalhoFilho PC, Figueiredo ACMG, et al. Periodontitis and respiratory diseases: a systematic review with meta-analysis. Oral Dis. 2019.

18. Moher D, Shamseer L, Clarke M, Ghersi D, Liberati A, Petticrew M, et al. Preferred reporting items for systematic review and meta-analysis protocols (PRISMA-P) 2015 statement. Syst Rev. 2015;1(4):1.

19. Sterne JAC, Savović J, Page MJ, Elbers RG, Blencowe NS, Boutron I, et al. RoB 2: a revised tool for assessing risk of bias in randomised trials. BMJ. 2019;28(366): 14898.

20. Sterne JA, Hernán MA, Reeves BC, Savović J, Berkman ND, Viswanathan M, et al. ROBINS-I: a tool for assessing risk of bias in non-randomised studies of interventions. BMJ. 355. 2016. https://www.bmj.com/content/355/bmj. i4919

21. Peters JL, Sutton AJ, Jones DR, Abrams KR, Rushton L. Contour-enhanced meta-analysis funnel plots help distinguish publication bias from other causes of asymmetry. J Clin Epidemiol. 2008;61(10):991-6.

22. Guyatt GH, Oxman AD, Schünemann HJ, Tugwell P, Knottnerus A. GRADE guidelines: a new series of articles in the Journal of Clinical Epidemiology. J Clin Epidemiol. 2011;64(4):380-2.

23. Schünemann HJ, Cuello C, AkI EA, Mustafa RA, Meerpohl JJ, Thayer $\mathrm{K}$, et al GRADE guidelines: 18. How ROBINS-I and other tools to assess risk of bias in nonrandomized studies should be used to rate the certainty of a body of evidence. J Clin Epidemiol. 2019;111:105-14.

24. Carrasco-Labra A, Brignardello-Petersen R, Santesso N, Neumann I, Mustafa RA, Mbuagbaw L, et al. Improving GRADE evidence tables part 1 : a randomized trial shows improved understanding of content in summary of findings tables with a new format. J Clin Epidemiol. 2016;74:7-18

25. Schünemann HJ, Brożek J, Guyatt G, Oxman A, editors. GRADE handbook for grading quality of evidence and strength of recommendations. Updated October 2013. The GRADE Working Group, 2013. 2013. guidelinedevelopment.org/handbook. 
26. DerSimonian R, Laird N. Meta-analysis in clinical trials. Control Clin Trials. 1986;7(3):177-88.

27. Das K, Das SJ, Sarma J. Effects of phase I periodontal therapy on the quality of life in COPD patients.

28. Agado BE, Crawford B, DeLaRosa J, Bowen DM, Peterson T, Neill K, et al. Effects of periodontal instrumentation on quality of life and illness in patients with chronic obstructive pulmonary disease: a pilot study. J Dent Hyg JDH. 2012;86(3):204-14.

29. Zhou X, Han J, Liu Z, Song Y, Wang Z, Sun Z. Effects of periodontal treatment on lung function and exacerbation frequency in patients with chronic obstructive pulmonary disease and chronic periodontitis: a. J Clin Periodontol. 2014;41(6):564-72.

30. Shen T-C, Chang P-Y, Lin C-L, Chen C-H, Tu C-Y, Hsia T-C, et al. Periodontal treatment reduces risk of adverse respiratory events in patients with chronic obstructive pulmonary disease. Med US. 2016;95(20):e3735.

31. Kucukcoskun M, Baser U, Oztekin G, Kiyan E, Yalcin F. Initial periodontal treatment for prevention of chronic obstructive pulmonary disease exacerbations. J Periodontol. 2013;84(7):863-70.

32. Liu Z, Zhang W, Zhang J, Zhou X, Zhang L, Song Y, et al. Oral hygiene, periodontal health and chronic obstructive pulmonary disease exacerbations. J Clin Periodontol. 2012;39(1):45-52.

33. Madalli R, Kheur S, Reddy M, Kheur M, Mahalle A. Assessment of role of Porphyromonas gingivalis as an aggravating factor for chronic obstructive pulmonary disease patients with periodontitis. Dent Hypotheses. 2016;7(3):100-6.

34. Armitage GC. Development of a classification system for periodontal diseases and conditions. Ann Periodontol. 1999;4(1):1-6.

35. Garcia-Rio F, Miravitlles M, Soriano JB, Muñoz L, Duran-Tauleria E, Sánchez $\mathrm{G}$, et al. Systemic inflammation in chronic obstructive pulmonary disease: a population-based study. Respir Res. 2010;11(1):63.

36. Van Eeden SF, Sin DD. Oxidative stress in chronic obstructive pulmonary disease: a lung and systemic process. Can Respir J J Can Thorac Soc. 2013;20(1):27-9.

37. Sinden NJ, Stockley RA. Systemic inflammation and comorbidity in COPD: a result of "overspill" of inflammatory mediators from the lungs? Rev Evid Thorax. 2010;65(10):930-6.

38. Yin $H, Y$ in $S, L i n ~ Q, X u Y, X u H$, Liu T. Prevalence of comorbidities in chronic obstructive pulmonary disease patients. Medicine (Baltimore). 2017;96(19). https://www.ncbi.nlm.nih.gov/pmc/articles/PMC5428602/

39. Chukkapalli SS, Easwaran M, Rivera-Kweh MF, Velsko IM, Ambadapadi S, Dai J, et al. Sequential colonization of periodontal pathogens in induction of periodontal disease and atherosclerosis in LDLRnull mice. Pathog Dis. 2017;75(1). https://www.ncbi.nlm.nih.gov/pmc/articles/PMC5353996/

40. Matthews JB, Wright HJ, Roberts A, Ling-Mountford N, Cooper PR, Chapple ILC. Neutrophil hyper-responsiveness in periodontitis. J Dent Res. 2007;86(8):718-22.
41. Irwin RS, Richardson ND. Side effects with inhaled corticosteroids: the physician's perception. Chest. 2006;130(1 Suppl):41S-53S.

42. Beeraka SS, Natarajan K, Patil R, Manne RK, Prathi VS, Kolaparthi VSK. Clinical and radiological assessment of effects of long-term corticosteroid therapy on oral health. Dent Res J. 2013;10(5):666-73.

43. Cardoso EM, Reis C, Manzanares-Céspedes MC. Chronic periodontitis, inflammatory cytokines, and interrelationship with other chronic diseases. Postgrad Med. 2018;130(1):98-104.

44. Usher AKH, Stockley RA. The link between chronic periodontitis and COPD: a common role for the neutrophil? BMC Med. 2013;13(11):241.

45. Zhou X, Wang J, Liu W, Huang X, Song Y, Wang Z, et al. Periodontal status and microbiologic pathogens in patients with chronic obstructive pulmonary disease and periodontitis: a case-control study. Int J Chron Obstruct Pulmon Dis. 2020;15:2071-9.

46. Tan L, Wang H, Li C, Pan Y. 16S rDNA-based metagenomic analysis of dental plaque and lung bacteria in patients with severe acute exacerbations of chronic obstructive pulmonary disease. J Periodontal Res. 2014;49(6):760-9.

47. Agado B, Bowen DM. Does the link between COPD and periodontitis affect dental hygiene treatment. Access. 2009;23(4):19-21.

48. Sapey E, Stockley RA. COPD exacerbations · 2: aetiology. Thorax. 2006;61(3):250-8

49. Sapey E, Yonel Z, Edgar R, Parmar S, Hobbins S, Newby P, et al. The clinical and inflammatory relationships between periodontitis and chronic obstructive pulmonary disease. J Clin Periodontol. 2020.

50. Pauwels RA, Buist AS, Calverley PM, Jenkins CR, Hurd SS. GOLD Scientific Committee. Global strategy for the diagnosis, management, and prevention of chronic obstructive pulmonary disease. NHLBI/WHO Global Initiative for Chronic Obstructive Lung Disease (GOLD) Workshop summary, Am J Respir Crit Care Med. 2001;163(5):1256-76.

51. Halpin DM, Miravitlles M, Metzdorf N, Celli B. Impact and prevention. of severe exacerbations of COPD: a review of the evidence. Int J Chron Obstruct Pulmon Dis. 2017;5(12):2891-908.

52. Dransfield MT, Kunisaki KM, Strand MJ, Anzueto A, Bhatt SP, Bowler RP, et al. Acute exacerbations and lung function loss in smokers with and without chronic obstructive pulmonary disease. Am J Respir Crit Care Med. 2017;195(3):324-30.

53. Amerio E, Mainas G, Petrova D, Giner Tarrida L, Nart J, Monje A. Compliance with supportive periodontal/peri-implant therapy: a systematic review. J Clin Periodontol. 2020;47(1):81-100.

\section{Publisher's Note}

Springer Nature remains neutral with regard to jurisdictional claims in published maps and institutional affiliations.

Ready to submit your research? Choose BMC and benefit from

- fast, convenient online submission

- thorough peer review by experienced researchers in your field

- rapid publication on acceptance

- support for research data, including large and complex data types

- gold Open Access which fosters wider collaboration and increased citations

- maximum visibility for your research: over $100 \mathrm{M}$ website views per year

At BMC, research is always in progress.

Learn more biomedcentral.com/submissions 